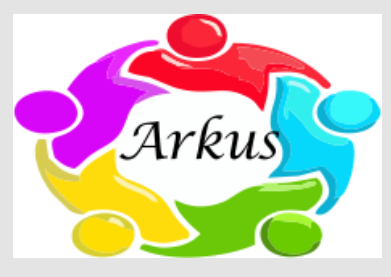

\title{
ARKUS
}

Journal Homepage:

https://hmpublisher.com/index.php/arkus

\section{New Face of Neuroinfection; Autoimmune Encephalitis}

\section{Andika Okparasta ${ }^{*}$}

${ }^{1}$ Department of Neurology, Faculty of Medicine, Sriwijaya University, Palembang, Indonesia

\section{A R T I C L E I N F O \\ Keywords: \\ Encephalitis \\ Autoimmune encephalitis \\ Corresponding author: \\ Andika Okparasta \\ E-mail address: \\ andikaokparasta@gmail.com}

The author has reviewed and approved the final version of the manuscript.

\begin{abstract}
A B S T R A C T
Encephalitis is an inflammatory process of the brain that attacks the central nervous system and can cause neurological dysfunction. Encephalitis is mostly caused by viruses, but in some cases the etiology is unknown. Autoimmune encephalitis occurs due to autoantibody interactions characterized by symptoms of central nervous system disorders. Clinical symptoms are quite specific, but there are criteria that must be met to make a diagnosis and additional investigations if needed. Treatment options for autoimmune encephalitis range from agents that suppress the immune system broadly to agents that specifically target antibody-mediated pathogenesis.
\end{abstract}

https://doi.org/10.37275/arkus.v3i1.121

\section{Introduction}

Encephalitis is a disease that attacks the central nervous system. Autoimmune encephalitis is a term used to describe a group of disorders characterized by the presence of symptoms of central nervous system disorders (limbic, extralimbic, basal ganglia, autonomic structures or more broadly) that occur due to autoantibody interactions at these locations. Autoimmune encephalitis includes several types of diseases with different pathophysiologies. The first is a classic paraneoplastic disorder associated with antibodies to intracellular antigens, eg anti-Hu. This disorder is strongly associated with cancer and involves a T-cell response that targets neurons but is not directly pathogenic. These autoantibodies crosslink with receptors and promote receptor internalization, which reduces receptor density on the neuron surface. This disorder is thought to be directly pathogenic, causing a reversible effect on neuronal synaptic function with relatively little neuronal death. ${ }^{1-3}$

\section{Clinical signs and symptoms}

The classic symptoms of autoimmune encephalitis are subacute (onset within days to weeks) and progressive, sometimes fluctuating, and cognitive changes. Memory, especially retention of new information can be impaired early in the course of the disease. The patient may then progress to coma. Each neuronal cell surface autoantibody is associated with a specific syndrome. For example, the manifestations of anti-NMDAR encephalitis can be categorized into eight groups: behavior and cognition, 
memory, speech, seizures, movement disorders, loss of consciousness, autonomic dysfunction, and central hypoventilation. Meanwhile, autoimmune encephalitis with antibodies to LGI1, GABABR, and AMPAR, usually presents with limbic syndrome symptoms, such as epilepsy, short-term memory loss, and psychiatric symptoms. Psychiatric manifestations are most common early in the course of autoimmune encephalitis. Manifestations include psychosis, aggression, inappropriate sexual behavior, panic attacks, compulsive behavior, euphoria, and fear. Symptoms can fluctuate rapidly. Abnormal movement can be a symptom of some types of autoimmune encephalitis. This movement resembles dystonia or chorea with writhing movements and abnormal postures of the extremities that remain. Sydenham's chorea is a well-known autoimmune movement disorder, which is thought to be triggered by a streptococcal infection and should be considered in children presenting with this symptom. Seizures are common in autoimmune encephalitis and can be a presenting symptom. Fasiobrachial dystonic seizures (FBDS) are brief seizures consisting of rapid jerks of the face and/or ipsilateral arm and shoulder. FBDS is characteristic of LGI1 autoimmunity and may precede other symptoms by weeks or months. ${ }^{4-8}$

\section{Conclusion}

Patients with AIE vary in their clinical presentation, severity, and comorbidities and respond differently to immunotherapy, so management must be individualized. Until now there are no guidelines and various treatments are given based on the clinical condition of the patient and the clinician's decision.

\section{References}

1. Kelley BP, Patel SC, Marin HL, Corrigan JJ, Mitsias PD, Griffith B. Autoimmune Encephalitis: Pathophysiology and Imaging Review of an Overlooked Diagnosis. Am J Neuroradiol. 2017; 38: 1070-8.

2. Venkatesan A, Tunkel AR, Bloch KC, Lauring AS, Sejvar J, Bitnun A, et al. Case Definitions, Diagnostic Algorithms, and Priorities in Encephalitis: Consensus Statement of the International Encephalitis Consortium. Clin Infect Dis. 2017; 57(8): 1114-28.

3. Graus F, Titulaer M, Balu R, Benseler S, Bien CG, Cellucci T. A Clinical Approach to Diagnosis of Autoimmune Encephalitis. Lancet Neurol. 2017; 15(4): 391-404.

4. Ropper AH, Dalmau J, Graus F. Antibody-Mediated Encephalitis. N Engl J Med. 2018; 379(9): 840-51.

5. Abrantes F, Toso FF, Povoas OG, Hoftberger R. Autoimmune encephalitis : a review of diagnosis and treatment. Arq Neuropsiquatry. 2017; 76(1): 41-9.

6. Shin Y, Lee S, Park K, Jung K, Jung K. Treatment strategies for autoimmune encephalitis. Journals sagepub. 2018; 11: 1-19.

7. Lee SK, Lee S. The Laboratory Diagnosis of Autoimmune Encephalitis. J Epilepsy Res. 2016; 6(2): 45-52.

8. Khadilkar S, Soni G, Patil S, Huchche A, Faldu H. Autoimmune Encephalitis : An update. J Assoc od Physicians India. 2017; 65(February): 62-9. 